\title{
Meta-analysis of transcriptomic responses to biotic and abiotic stress in tomato
}

\author{
Elham Ashrafi-Dehkordi ${ }^{1}$, Abbas Alemzadeh ${ }^{\text {Corresp., }}{ }^{1}$, Nobukazu Tanaka ${ }^{2}$ \\ 1 Department of Crop Production and Plant breeding, School of Agriculture, Shiraz University, Shiraz, Iran \\ ${ }^{2}$ Center of Gene Science, Hiroshima University, Kagamiyama, Higashi-Hiroshima, Hiroshima, Japan \\ Corresponding Author: Abbas Alemzadeh \\ Email address: alemzadeh@shirazu.ac.ir
}

A wide range of biotic and abiotic stresses adversely affects plant growth and productivity worldwide. The study of individual genes cannot consider as an effective approach for the understanding of tolerance mechanisms since these stresses frequently, often in combination and a large number of genes involved in these mechanisms. The availability of high-throughput genomic data has enabled the discovery of the role of transcription factors in regulatory networks. A meta-analysis of biotic and abiotic stress responses was performed by analyzing a total of 391 microarray samples from 23 different experiments and 2336 differentially expressed genes involved in multiple stresses were identified. We identified 1862 genes differentially regulated in response to biotic stresses was much greater than that regulated by abiotic stresses, 835 genes, and found $15.4 \%$ or 361 differentially expressed genes with the conserved expression between abiotic and biotic stresses. The greatest percent of genes related to the cellular process ( $>76 \%$ genes), metabolic process ( $>76 \%$ genes) and response to stimulus ( $>50 \%$ ). About $4.2 \%$ of genes involved in biotic and abiotic stress responses belonged to the transcription factor families. We identified several genes, which encode transcription factors that play an important role in abiotic and biotic stress responses. These proteins included JERF1, SIGRAS6, MYB48, SIERF4, EIL2, protein LHY, SIERF1, WRKY 26, bZIP transcription factor, ICE1-like, pti6, EIL3 and WRKY 11. Six of these proteins, JERF1, MYB48, protein LHY, EIL3, EIL2 and SIGRAS6 play central roles in these mechanisms. This research promoted a new approach to clarify the expression profiles of various genes under different conditions in plants, detected common genes from differentially regulated in response to these conditions and introduced them as candidate genes for improving plant tolerance through genetic engineering approach. 
1 Meta-analysis of transcriptomic responses to biotic and abiotic stress in

2

3

$4{ }^{1}$ Department of Crop Production and Plant Breeding, School of Agriculture, Shiraz University,

5 Shiraz, Iran

$6{ }^{2}$ Center of Gene Science, Hiroshima University, 1-4-2 Kagamiyama, Higashi-Hiroshima,

7 Hiroshima, Japan

8

9 Corresponding author

10 Abbas Alemzadeh

11

12 alemzadeh@shirazu.ac.ir

13

14

15

16

17

18

19

20

21

22

23

tomato

Elham Ashrafi-Dehkordi ${ }^{1}$, Abbas Alemzadeh $^{1}$ and NobuKazu Tanaka ${ }^{2}$

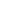

8

\section{Corresponding author}

1

1

rom

14

15

16

17

9 
24 ABSTRACT

25 A wide range of biotic and abiotic stresses adversely affects plant growth and productivity worldwide. The study of individual genes cannot consider as an effective approach for the understanding of tolerance mechanisms since these stresses frequently, often in combination and a large number of genes involved in these mechanisms. The availability of high-throughput genomic data has enabled the discovery of the role of transcription factors in regulatory networks. A meta-analysis of biotic and abiotic stress responses was performed by analyzing a total of 391 microarray samples from 23 different experiments and 2336 differentially expressed genes involved in multiple stresses were identified. We identified 1862 genes differentially regulated in response to biotic stresses was much greater than that regulated by abiotic stresses, 835 genes, and found $15.4 \%$ or 361 differentially expressed genes with the conserved expression between abiotic and biotic stresses. The greatest percent of genes related to the cellular process ( $>76 \%$ genes), metabolic process ( $>76 \%$ genes) and response to stimulus $(>50 \%)$. About $4.2 \%$ of genes involved in biotic and abiotic stress responses belonged to the transcription factor families. We identified several genes, which encode transcription factors that play an important role in abiotic and biotic stress responses. These proteins included JERF1, SIGRAS6, MYB48, SIERF4, EIL2, protein LHY, SlERF1, WRKY 26, bZIP transcription factor, ICE1-like, pti6, EIL3 and WRKY 11. Six of these proteins, JERF1, MYB48, protein LHY, EIL3, EIL2 and SIGRAS6 play central roles in these mechanisms. This research promoted a new approach to clarify the expression profiles of various genes under different conditions in plants, detected common genes from differentially regulated in response to these conditions and introduced them as candidate genes for improving plant tolerance through genetic engineering approach. 
48

49

50

51

52

53

54

55

56

57

58

\section{INTRODUCTION}

As sessile organisms, plants constantly encounter a wide range of stresses that negatively affect the yield production, survival and growth of plants worldwide (Sharma et al., 2013). The stresses are divided into two broad categories, abiotic stresses (AS) including a variety of adverse environmental conditions, (i.e. drought, submergence, salinity, heavy metal contamination, nutrient deficiency, light and extreme temperatures) and biotic stresses (BS) caused by attack of living organisms, (i.e. bacteria, viruses, fungi and nematodes as well as other plants) (Rastgoo \& Alemzadeh, 2011; Liu et al., 2014). To survive under stress conditions, complex mechanisms have evolved in plants to perceive signals from their environment, transmit through signal transduction pathways and respond to the various stresses that each of which may involve several hundred proteins (Fujita et al., 2006; Rastgoo, Alemzadeh \& Afsharifar, 2011; Atkinson, Lilley \& Urwin, 2013; Sharma et al., 2013, Sami \& Alemzadeh, 2016). Functional molecular studies have figured out interactions between biotic and abiotic stresses signaling components with the identification a large number of genes, which are regulated by various types of transcription factors (TFs) (Kissoudis et al., 2016). TFs are proteins with critical roles in the control temporal and spatial gene expression during defense response that many of them are induced by more than one stress (Liu et al., 2014; Shaik \& Ramakrishna, 2014; Prasad et al., 2016).

The better understanding of plant stress response mechanisms under various stresses can give us a higher view of how to improve the worldwide food production (Sharma et al., 2013). It is necessary to learn more about molecular mechanisms of transcription factors, MAPKs and ROS signal pathways, and hormone signaling, and study of genes encoding regulatory molecules, especially transcription factors, can be more efficient than single-function genes, because TFs enable to regulate various genes to improve plant tolerance against stresses (Seki et al., 2002). 
71 The bioinformatic approach is an emerging and rapidly developing field which allows

72 researchers to analyze biological data (Khatri \& Drăghici, 2005; Chang et al., 2013; Zinati,

73 Alemzadeh \& KayvanJoo 2016). Recent advances in genome-wide transcriptome analysis

74 methods, such as RNA sequencing and microarrays, enable researchers simultaneously to study

75 the expression of thousands of genes and their coexpression partners under various stresses

76 (Khatri \& Drăghici, 2005; Sharma et al., 2013; Rest et al., 2016). Meta-analysis can be used to

77 estimate differential gene expression between normal and stress conditions and find out some

78 genes that their products are key molecules in response to the stress (Tseng, Ghosh \& Feingold,

79 2012; Shaar-Moshe, Hübner \& Peleg, 2015).

80 Today, with recent advances in high-throughput technology, a large number of biological data

81 has become accessible via online resources, and we are now able to obtain results that more

82 reliable by combining information from multiple sources. In this way, statistical methods can be

83 used as useful and powerful tools to identify suitable candidate genes for test under experimental

84 conditions to use in plant breeding programs. The combination of $P$-values is a common method

85 in microarray meta-analysis and recently used in many studies (Tseng, Ghosh \& Feingold, 2012).

86 A meta-analysis of microarray using 13 experiments querying cytokinin-regulated gene

87 expression changes in Arabidopsis combined with empirically defined filtering criteria identified

88 a set of 226 genes differentially regulated by cytokinin (Bhargava et al., 2013). In another

89 research project, a meta-analysis of ten studies identified 4015 genes showing significant

90 differential expression in response to water limitation compared to control conditions in

91 Arabidopsis (Rest et al., 2016).

92 Moreover, combining gene expression information across species can improve the ability to

93 identify core gene sets with high evolutionary conservation. For example, a new cross-species 
94 meta-analysis of progressive drought stress at the reproductive stage was developed using 95 Arabidopsis, rice, wheat and barley. Based on this dataset, shared important genes and metabolic 96 pathways involved in whole plant adaptation to progressive drought stress across-species were 97 identified (Shaar-Moshe, Hübner \& Peleg, 2015). In this study, 225 differentially expressed genes (DEGs) shared across studies and taxa were identified and gene ontology enrichment and pathway analyses revealed that the shared genes were classified into functional categories involved predominantly in metabolic processes, regulatory function and response to stimulus (Shaar-Moshe, Hübner \& Peleg, 2015).

Tomato (Solanum lycopersicum) is one of the most important food crops belongs to Solanaceae family, one of the largest and most important families of flowering plants. In addition, the tomato is a suitable model system to study environmental signal transduction components involved in biotic and abiotic stresses (Knapp et al., 2004; Arie et al., 2007). However, there is no a largescale transcriptome analysis via meta-analysis of stress response studies in tomato to detect proteins, especially transcription factors, involved in various stresses.

In this study, for the first time we performed a large scale comparative transcriptome analysis via meta-analysis of stress response studies in tomato using microarray gene expression data implemented on a single platform (Affymetrix Tomato Genome Array). The goal of this metaanalysis was to detect DEGs involved in biotic and abiotic stresses and detect DEGs shared by

112 both stresses. In addition, we focused on the identification of transcription factors have magnitude effects on the plant cells via up- and down- regulation of various genes under stress conditions. Other analysis methods, such as gene ontology (GO) and Network analysis, were used to explain the complexity of tomato response to abiotic and biotic stresses. We also 116 discussed the functions of the identified transcription factors in stress response. 
METHODS

\section{Selection microarray studies}

120 In this study, a meta-analysis of stress response was performed in tomato using publicly

121 available microarray gene expression data implemented on a single platform (Affymetrix

122 Tomato Genome Array). Expression data of tomato plants exposed to biotic and abiotic stresses

123 were combined. The raw expression data of various experiments were obtained from the Array

124 Express from EBI (https://www.ebi.ac.uk/arrayexpress/).

\section{Identification of differentially expressed genes}

126 Microarray expression data from each source study was pre-processed separately as individual 127 datasets. The raw data were normalized using Robust Multi-array Average (RMA) background correction and quantile normalization. The data were processed in the $\mathrm{R}$ program. Meta-analysis of AS and BS was performed separately to identify DEGs involved in stress conditions. Genes that showed significant $P$-value (FDR of 5\%), meaning that an estimated 5 percent of the DEGs are false positive, were considered as DEGs (Campain \& Yang, 2010; Chang et al., 2013). Two methods of the combination of $P$-value have used: Fisher's method and maxP (Wilkinson, 1951; Fisher, 1992). The Fisher's combined probability test sums the logarithm-transformed $P$-values obtained from individual studies, under the null hypothesis, and follows a chi-squared distribution with $2 \mathrm{k}$ degrees of freedom, where $\mathrm{k}$ is the number of studies being combined

136 (Rhodes et al., 2002). A very small $P$-value in just one study can be adequate to make statistical

137 significance, even if the same genes are not significant in any other study. The maxP method 138 takes maximum $P$-values across studies. It follows a beta distribution with parameters $\mathrm{K}$ (number 139 of transcriptomic studies which are combined for a meta-analysis where each study contains G 
140 genes) and 1. This method targets on DEGs that have small $p$-values in all studies. (Chang et al.,

141 2013; Song \& Tseng, 2014).

142 Gene Analysis

143 The genes selected by meta-analysis were further analyzed and characterized. In the first

144 instance, the results were compared with the plant genes in Plant Transcription factor \& Protein

145 Kinase Identifier and Classifier, iTAK (http://bioinfo.bti.cornell.edu/cgi-bin/itak/index.cgi) and

146 Plant Transcription Factor, PlantTFDB (http://plntfdb.bio.uni-potsdam.de/v3.0/) databases. The

147 list includes 1845 genes of tomato which encoding the TFs that directly or indirectly involved in

148 signaling pathway and response to abiotic and biotic stresses. Venn's diagrams

149 (http://bioinfogp.cnb.csic.es/tools/venny/) were used to represent co-occurrence of DEGs. Gene

150 Ontology (GO) enrichment analysis of the DEGs (such as identifying biological processes) was

151 conducted using the AgriGO platform (Du et al., 2010).

152 Network analysis

153 The Search Tool for the Retrieval of Interacting Genes/Proteins, STRING 10.5

154 (http://www.mybiosoftware.com/string-9-0-search-tool-retrieval-interacting-genesproteins.html)

155 (Szklarczyk et al., 2014) database was used to figure out all functional relations between the

156 genes and their occurrence patterns across various genomes. The database makes relations based

157 on several lines of evidences: The empirical evidence from protein-protein interaction assays,

158 co-expression data from NCBI Gene Expression Omnibus (GEO) database, the extraction of

159 information from other databases, coexistence of the genes in the same organisms, conserved

160 gene neighborhood in known genomes, gene fusion events, pathway annotation from other

161 resources such as Kyoto Encyclopedia of Genes and Genomes (KEGG) or Gene Ontology

162 databases, and automated text-mining tools (Szklarczyk et al., 2014). STRING calculates a 
163 confidence value for those interactions according to the pieces of evidences from above 0.4 to

164 above 0.9 , as the medium to highest score, respectively.

165

166 RESULTS AND DISCUSSION

167 Identification of genes involved in biotic and abiotic stresses

168 After searching in the database, microarray data from 23 different experiments were selected and 169 analyzed. To obtain a global analysis, 391 microarray samples including 213 AS from different 170 categories: drought, heat, salinity, light, hormone, mineral and heavy metals 171 deficiencies/toxicities and $178 \mathrm{BS}$ that caused by viruses, nematode, fungi, bacteria and pests in 172 tomato (wild type and transgenic) were chosen (Table S1). We found a total of 1862 genes 173 differentially regulated in response to biotic stresses, was far greater than that induced by abiotic 174 stresses, 835 genes, with a false discovery rate $($ FDR $) \leq 0.05$ by Fisher's statistical method 175 (Table S1-S3). We also figured out $15.4 \%$ or 361 DEGs with the conserved expression between 176 AS and BS by Fisher's statistical method, suggesting that these genes and their associated cell177 signaling pathways are regulated in a similar way in a wide range of stresses (Fig. 1A) (Table 178 S5). Using maxP method, a total of 14 genes differentially regulated in response to abiotic 179 stresses and a total of 220 genes differentially regulated in response to biotic stresses were found. 180 We figured out $0.9 \%$ or 2 DEGs (LES.1399.1 and LES.3969.1) with the conserved expression 181 between AS and BS by maxP method (Fig. 1A) (Table S2-S4). These methods used in other 182 studies to identify important genes in different physiological processes in other plants. Shaar183 Moshe Hübner and Peleg (2015) identified 225 differentially expressed genes across-species by using 17 microarray experiments of drought stress at the reproductive stage of Arabidopsis, rice, wheat and barley. Fisher's and maxP methods are two popular microarray meta-analysis methods 
186 used in the literature that have different strength for detecting different types of DEGs (Tseng,

187 Ghosh \& Feingold, 2012; Wang et al., 2012). Our results also showed that the two used methods

188 detected different sets of DEGs, suggesting different assumptions behind the methods. Among

189 these methods, Fisher's method is more popular in the microarray meta-analysis studies (Wang et 190 al., 2012) and hence, we were more interested in detecting DEGs across all studies through

191 Fisher's statistical method.

\section{Gene ontology}

194 We focused on the 361 DEGs common to both AS and BS and subjected them to Gene Ontology 195 (GO) and network analysis to explore other possible functions of them. GO enrichment analysis 196 of the DEGs showed that shared DEGs represented genes involved in main biological and 197 cellular processes. Among them, the greatest percentage of genes involved in the cellular process 198 ( $>76 \%$ genes $)$, metabolic process ( $>76 \%$ genes) and response to stimulus $(>50 \%)$. One of largest 199 functional group of DEGs was associated with metabolic processes (e.g. carbohydrate metabolic 200 process, lipid metabolic process, protein metabolic process, nucleobase, nucleoside, nucleotide 201 and nucleic acid metabolic process, cellular amino acid and derivative metabolic process and 202 regulation of primary metabolic process).

In addition, another large functional group of DEGs was associated with cellular processes (e.g. cellular metabolic process, regulation of cellular process and cellular response to stimulus), the show a significant rearrangement in plant metabolism as part of stress adaptation (Shaar-Moshe, Hübner \& Peleg, 2015).

207 On the other hand, the lowest percentage of genes involved in positive regulation of biological 208 process (5\%) (Fig. 2) (Table S6). The most significant GO terms for cellular process were 
209 cellular metabolic process (FDR: 1.8E-37), organic acid metabolic process (FDR: 9.2E-29),

210 cellular ketone metabolic process (FDR: 1.2E-28) in metabolic process were primary metabolic

211 process (FDR: 7.8E-39), cellular metabolic process (FDR: 1.8E-37) and organic acid metabolic

212 process (FDR: 9.2E-29). Moreover, in response to stimulus were response to abiotic stimulus

213 (FDR: 3.4E-40), response to biotic stimulus (FDR: 9.4E-26), and response to stress (FDR: 9.4E-

214 26). Some of these genes possess important roles in DEGs shared by AS and BS networks (Table

215 S7). A genome-wide transcriptional analysis was performed just for Fe deficiency and was

216 identified 97 DEGs by comparing Fe deficient and Fe-sufficient in roots of tomato. These

217 transcripts are related to the physiological responses to the nutrient stress resulting in an

218 improved iron uptake, including regulatory aspects, translocation, root morphological

219 modification, and adaptation in primary metabolic pathways, such as glycolysis and TCA cycle

220 (Zamboni et al., 2012). In another research was showing that the largest functional groups of

221 DEGs was associated respectively to metabolic processes (e.g. amino acid and carbohydrate

222 metabolism), regulator function (e.g. protein degradation and transcription) and response to the

223 stimulus that identified by the research of Shaar-Moshe Hübner and Peleg (2015).

\section{Network analysis}

225 Network analysis using STRING 10.5 identified the connections among DEGs common to AS 226 and BS (Fig. 3) (Table S7). In addotion, some genes that possess an important role in the three

227 biological pathways; cellular process, metabolic process, response to stimulus were detected. For 228 example, Solyc02g090890.2.1 (ZE: plays an important role in resistance to stresses, seed 229 development and dormancy), Solyc04g049350.2.1 (CS1: biosynthesis of aromatic amino acids), 230 Solyc07g052480.2.1 (LOC544276: involved in storage lipid mobilization during the growth of 231 higher plant seedling), Solyc07g049530.2.1 (ACO1: involved in ethylene biosynthesis via S232 adenosyl-L-methionine), Solyc01g099630.2.1 (XTH1: an essential component of the primary cell 233 wall, and thereby participates in cell wall construction of growing tissues), Solyc05g055230.1.1 234 (RPS17: ribosomal small subunit assembly), Solyc12g098350.1.1 (infA: no specific function has 
235 so far been attributed to this initiation factor; however, it seems to stimulate more or less all the

236 activities of the other two initiation factors, IF-2 and IF-3), Solyc02g082920.2.1 (CHI3: defense

237 against chitin containing fungal pathogens), Solyc06g082600.2.1 (UBC3: mediates the selective

238 degradation of abnormal and short-lived proteins), Solyc01g096480.2.1 (LOC543657: probable

239 thiol-disulfide oxidoreductase that may play a role in proper chloroplast development and 240 negatively regulates Cf-9/Avr9-mediated cell death and defense responses), Solyc07g047850.2.1

241 ( $C A B 4$ : it receives and transfers excitation energy to photosystems).

242 The results of network analysis also showed some of the transcription factors have connections 243 to other molecules. Based on pieces of literatures reviewing, detected transcription factors in this 244 study, JERF1, EIL3, EIL2, SIGRAS6, Protein LHY and MYB 48 affected by a wide range of 245 stresses and possibly play essential and significant roles in multiple stress responses which is in 246 agreement with their functional roles in biotic and abiotic stress responses. Expression of JERF1 247 was induced by salt, ethylene, MeJA and abscisic acid (ABA) treatments in tomato that may be 248 pointed to a central role for JERF1 in different signal transduction pathways (Zhang et al., 2004).

249 It has been also reported that JERF1 activates ABA biosynthesis related genes such as NtSDR 250 (short-chain dehydrogenase/reductase) and enhanced cold and salt stress tolerance in transgenic 251 tobacco (Wu et al., 2007; Ouyang et al., 2007). It showed that the expression of EIL2 and EIL3 252 was induced by ethylene and salinity treatments (Ying et al., 2004) and SlGRAS6 is one of the 253 most important members of the tomato GRAS family in disease resistance and mechanical stress 254 (Mayrose et al., 2006). Different studies have shown the large family of MYB has essential roles 255 in plant growth, development, primary and secondary metabolism and response to BS and AS 256 (Oh, Park \& Han, 2003; Van et al., 2008; Dou et al., 2016). It also reported that LHY controls 257 the expression of a large number of ABA-responsive genes by binding directly to their promoters 258 (Huang et al., 2007). These results support that the detected transcription factors in our study 259 might play an important role in biotic and abiotic stress responses.

260

Identification of transcription factors involved in biotic and abiotic stresses

262

In this research, because of the importance of TFs as a powerful tool for the manipulation of complex metabolic pathways, we have tried to identify genes encoding them. According to the PlantTFDB, 1845 genes from tomato encoding TFs were identified and classified into 58 
265 families that directly or indirectly involved in signaling and response to AS and BS (Table S8).

266 Based on our results, from the 1862 genes response to biotic stresses selected by Fisher's 267 statistical method, 93 genes (5\%) encode TFs (Fig.1C) (Table S9). ERF family has the greatest 268 number of genes (18 genes), and C3H, FAR1, GATA, LBD, MIKC-MADS, PHD, SLP protein, 269 TALE, TIFE and YABBY families have the lowest number of genes (each family has only one 270 gene) (Table 1). From the 835 genes response to abiotic stresses selected by Fisher's statistical 271 method, 31 of those (3.7\%) encode TFs (Fig. 1B) (Table S10). ERF and NAC families have the 272 greatest number of genes (5 genes), and ARF, BBR-BPC, GRAS, NF-NY and VOZ families 273 have the lowest number of genes (each family has only one gene) (Table 1).

274 Since TFs are the key regulators of plant growth, development and metabolisms via up- and 275 down- regulation of various genes, identification of the genes encoding these proteins and transferring them into plants to improve their stress tolerance would be interesting. These TFs 277 (JERF1, Pti6, SlERF1, SlERF4, SlGRAS6, MYB48, protein LHY, EIL2, EIL3, WRKY 11, WRKY 26, ICE1-like and bZIP transcription factor) can be used as candidate genes in molecular breeding programs. Because, these TFs are common to abiotic and biotic stresses and also, our results indicate they are being affected by a wide range of stresses. In addition, they possibly play essential and significant roles in multiple stress responses which is in agreement with their functional roles in biotic and abiotic stress responses (Gu et al., 2002; Ying et al., 2004; Zhang et al., 2004; Borrás-Hidalgo et al., 2005; Mayrose et al., 2006; Wu et al., 2007; Yáñez et al., 2009; Zhang et al., 2010; Sharma et al., 2010; Chen et al., 2012; Kim, Stork \& Mudgett, 2013; Feng et proteins have central roles in biotic and abiotic stress responses (Fig. 3). The two-way Venn 
287 diagram indicated that the number of common TFs between biotic and abiotic stresses was 13

288 while 18 and 80 TFs were uniquely identified in abiotic and biotic stresses, respectively (Fig. 4).

\section{ERF family}

290 The ERF (Ethylene-responsive transcription factors) has been shown to play a key role during 291 plant development and stress tolerance mechanisms. Overexpression of ERF family genes in 292 different plant species such as Arabidopsis, tobacco, rice and tomato has been shown to increase tolerance to a wide range of biotic and abiotic stresses (Sharma et al., 2010). The tomato has 112 AP2/ERF superfamily genes (Sharma et al., 2010; Sharma et al., 2013). Our results showed that 4 of 13 transcription factors shared between AS and BS, were belong to ERF family, including JERF1, SIERF4, SIERF1 and pti6 (Table 2).

JERF1, Jasmonate Ethylene Response Factor 1, has critical roles in plant abiotic stress responses, such as salinity, low temperature, dehydration and various biotic stresses in different plant species (Zhang et al., 2004; Wu et al., 2007; Zhang et al., 2010). It has been shown that the expression of JERF1 in tomato was induced by salt, ethylene, MeJA and abscisic acid (ABA) treatments that may be pointed to a central role for JERF1 in different signal transduction pathways (Zhang et al., 2004). Overexpression of JERF1 enhanced the tolerance of transgenic tobacco and rice plants to high salinity, low temperature, osmotic stress and pathogen attack by regulating the expression of downstream stress-responsive genes and a number of pathogenesisrelated (PR) genes due to binding to DRE/CRT and GCC-box cis-elements ( Wu et al., 2007; Zhang et al., 2010). Expressing JERF1 in tobacco induced expression of GCC box-containing genes such as $C H N 50, \operatorname{Prb}-1 b, G L A, N t S D R$ and osmotin that subsequently resulted in enhanced tolerance to salt stress and low temperature (Zhang et al., 2004; Wu et al., 2007). JERF1 upregulated the expression of genes related to osmolyte synthesis such as OsP5CS and OsSPDS2, 
310 which, has increased the production of osmolytes. Also it induced the expression of $O s A B A 2$ and

311 Os03g0810800 genes (containing DRE element in their promoter regions), which encode two

312 key enzymes involved in ABA biosynthesis and increased the synthesis of ABA in transgenic

313 rice that resulted in elevated tolerance to drought (Zhang et al., 2010). JERF1 may also be

314 located downstream of EIN3 in the ethylene signaling pathway (Zhang et al., 2004). It has been

315 previously reported that SlERF4, an ERF in tomato, is a XopD, a type III secretion effector from

316 Xanthomonas campestris, destabilize SlERF4 and this task has been proposed to promote

317 bacterial growth (Kim, Stork \& Mudgett, 2013). SlERF4 reduces ethylene production and, in 318 turn, limits disease symptom development (Kim, Stork \& Mudgett, 2013) (Table 2). Pti6

319 belonged to the ERF family in the plant that binds specifically to the GCC-box cis element

320 present in the promoter regions of many PR genes and induced the expression of a wide range of

321 these genes that have key roles in the plant defense. It also showed that the expression of 322 jasmonic acid- and ethylene-regulated genes, such as PR3, PR4, PDF1.2, and Thi2.1, was 323 differently being affected by Pti4 or other similar genes, Pti5, or Pti6 (Gu et al., 2002;

324 Chakravarthy et al., 2003) (Table 2). The gene encoding SlERF1 was another detected gene that 325 probably has an important role in response to various stresses. It has been shown that the 326 overexpression of SlERF1 in Arabidopsis can confer resistance to necrotrophic fungi like 327 Plectosphaerella cucumerina and Botrytis cinerea (Mayrose et al., 2006) (Table 2).

328 GRAS family

329 SIGRAS6 was another identified transcription factor that is one of the most important members 330 of tomato GRAS family in disease resistance and mechanical stress (Mayrose et al., 2006).

331 SIGRAS6 is required for tomato disease resistance to the bacterial pathogen Pseudomonas 332 syringae pv. tomato. It has been shown that the suppression of SlGRAS6 gene expression through 
333 virus-induced gene silencing reduced resistance of tomato to P. syringae pv. tomato (Mayrose et

334 al., 2006) (Table 2).

\section{MYB family and MYB-related family}

336 The MYB is a large TF family in plants that have essential roles in plant growth, development,

337 primary and secondary metabolism and response to BS and AS (Oh, Park \& Han, 2003; Van et 338 al., 2008; Dou et al., 2016). Most of MYB genes involved in response to various abiotic stresses,

339 belong to the R2R3-type group. With the comparison of OsMYB48-1 expression with other MYB

340 proteins, a special role for this gene in response to the abiotic stresses in rice can be suggested. It

341 has been shown that its expression was strongly induced by $\mathrm{ABA}, \mathrm{H}_{2} \mathrm{O}_{2}, \mathrm{PEG}$, and dehydration

342 stresses, while slightly induced by salt and cold stresses (Xiong et al., 2014). It was reported that

343 the overexpression of $O S M Y B 48-1$ regulated the expression level of some stress responsive344 genes, such as $R A B 21, R A B 16 D, R A B 16 C$ and $L E A 3$ under drought stress conditions (Xiong et 345 al., 2014) (Table 2). The LHY (LATE ELONGATED HYPOCOTYL) transcription factor plays 346 a critical role in the regulation of low temperature stress response via controlling expression of

$347 C B F$ 1, 2 and 3, COR27 and COL1 genes and contribute to plant defense through the circadian 348 regulation of stomatal aperture (Grundy, Stoker \& Carré, 2015; Seo \& Mas, 2015). It was also 349 shown that LHY controls the expression of a large number of ABA-responsive genes by binding 350 directly to their promoters (Huang et al., 2007).

\section{EIL family}

352 It has been shown that the expression of EIL2 and EIL3, which encode transcription factors in 353 ethylene signal transduction, was induced by ethylene and salinity treatments (Ying et al., 2004). 
354 It also reported that its ortholog in tobacco is required for resistance against Peronospora

355 hyoscyami (Borrás-Hidalgo et al., 2006) (Table 2).

\section{WRKY family}

357 Different studies have shown that the members of WRKY family contribute multiple biological

358 processes such as plant growth and development. They also have important roles in a wide range

359 of biotic and abiotic stresses such as drought, salt, invasion of pathogens, implying that the

360 members of this family may be putative regulators in response to various BS and AS (Huang et

361 al., 2012; Li, Luan \& Jin, 2012). Two of 13 transcription factors, WRKY11 and WRKY26, which

362 involved in both AS and BS belong to the WRKY family (Table 2). In other plant species, it also

363 showed that the members of this family have a key role in abiotic and biotic stress responses ( $L i$

364 et al., 2011; Chen et al., 2012). It was reported that OsWRKY11 involved in drought and heat

365 tolerance in rice ( $W u$ et al., 2009). It was shown that, in Arabidopsis thaliana, WRKY26

366 positively regulates the cooperation between heat shock proteins and ethylene-activated signaling

367 pathways that mediate responses to heat stress ( $\mathrm{Li}$ et al., 2011). It has also been shown that the

368 promoters of some heat tolerance-related genes including $H_{s p}$ and $H s f$ genes such as $H_{s f} A 2$,

$369 H s f B 1, H s p 101$, and MBF1c contain W-box sequences that are recognized by WRKY proteins

370 (Li et al., 2011).

\section{ICE1-like bHLH family}

372 ICE1-like (inducer of CBF expression 1) encodes a MYC-type basic helix-loop-helix (bHLH)

373 transcription factor (Chinnusamy et al., 2003). ICE1 is an important inducer of CBF3/DREB1A

374 which regulates cold stress tolerance (Chinnusamy et al., 2003; Huang et al., 2015; Feng et al.,

375 2013) (Table 2). In Arabidopsis, icel mutants showed decreased cold and chilling tolerance, 
376 whereas ICE1 overexpression in Arabidopsis increased the cold tolerance of transgenic plants

377 (Chinnusamy et al., 2003). Furthermore, osmotic and salt tolerance were also much markedly

378 increased in transgenic tobacco carrying SIICE1 from tomato by increasing the expression level

379 of DREB1/CBF and their target genes (Feng et al., 2013) (Table 2).

380

381

382

383

384

385

386

387

388

389

390

391

392

393

394

395

396

397

398

\section{bZIP family}

Transcription factors of the basic leucine zipper (bZIP) family regulate various biological processes in plant growth and development, including morphogenesis and seed formation, as well as in abiotic and biotic stress responses by binding to promoters of specific target genes to up- or down- regulate their expression ( $\mathrm{Li}$ et al., 2015). WRKY transcription factors, basic leucine zipper domain (bZIP) and MYB transcription factors involved in plant defense against pathogens (Alves et al., 2013) (Table 2). Transcription factor bZIP11 is a member of the S1 class of the bZIP family, and it was shown that bZIP11 mRNA translation is repressed in response to sucrose (Hummel et al., 2009). It has been shown that sucrose repression of bZIP11 mRNA translation depends on the presence of the 5'-leader sequence upstream of bZIP11 gene (Hummel et al., 2009). It has also been shown that sucrose signaling has an important role in the translational control of bZIP11 in response to stress conditions (Hummel et al., 2009).

These reports implied that these transcription factors are important regulators of biological processes and may be useful in the breeding of plants to improve their tolerance against adverse stresses. The presence or absence of the transcription factors across various plant species and other organisms including bacteria, archaea and other eukaryotes is shown in figure 5. A high homology was observed for all transcription factors except protein LHY between Solanum lycopersicum and Solanum tuberosum and the highest percentage of similarity was found between Vitis vinifera, Populus trichocarpa and Glycine max. The results showed that GRAS6 is 
399 the only TF present in bacteria with a low similarity to that of tomato GRAS6. Just three of the 400 identified TFs, ICE1-like, protein LHY and MYB48, are present in opisthokonta that the 401 homology between MYB48 and those of opisthokonata was the highest. MYB48 is the only 402 protein, which is present in all organisms except bacteria and archaea (Fig. 5). It has been shown 403 that MYB is one of the largest and most diverse transcription factor families, which are the key 404 factors in regulatory networks controlling metabolism, development and responses to BS and AS 405 (Roy, 2016). The members of MYB family found in all eukaryotes and molecular studies in 406 Arabidopsis has been shown the role of this family in the epigenetic regulation of stress 407 responses in plants (Roy, 2016). The results showed MYB48 as one of the members of MYB 408 family possesses an important role in the network of abiotic and biotic responses (Fig. 3). In 409 addition to MYB48, three other TFs, WRKY11, WRKY26 and protein LHY, were observed in Amoebozoa with low similarity to those of tomato (Fig. 5). As well as Amoebozoa, the WRKYs were observed in Giardia lamblia, also known as Giardia intestinalis (Fig. 5). It was previously 412 thought that WRKYs are specific to plants and algae (Eulgem \& Somssich, 2007) but recent 413 studies show their presence in human protozoan parasite G. lamblia and D. discoideum (slime 414 mold) (Wu et al., 2005). It has been reported that WRKYs have also important roles in other physiological processes. It was shown that the expression of genes encoding proteins of cell wall 416 increased, when a WRKY-like gene overexpressed in G. lamblia (Pan et al., 2009).

417 The results showed that protein LHY present in all eukaryotes except G. lamblia, Trichomonas 418 vaginalis and Trypanosomatidea (Fig. 5) and also suggested a central role for this protein in the 419 network of abiotic and biotic responses (Fig. 3). The results of other studies also support an 420 important role for this protein and suggested that this protein involved in circadian clock control 421 (Lu et al., 2009). 


\section{CONCLUSION}

424 Transcriptome-wide studies by meta-analysis allowed us to narrow down DEGs to a small 425 number. DEGs represent the genes in different networks and signaling pathways that enable us to 426 figure out which genes play the major role in various stresses and determine the overlap between 427 biotic and abiotic stress response pathways.

428 This study was designed to identify genes, which, have important roles in abiotic and biotic 429 tolerance mechanisms in tomato and focused on those shared by both stresses. The novelty of the 430 work presented here is that a large meta-analysis was used to identify important genes in tomato 431 as a model of Solanaceae crops. Meta-analysis has been previously used to figure out important 432 genes that play critical roles in response to biotic or abiotic stresses in other plants such as 433 Arabidopsis (Shaar-Moshe, Hübner \& Peleg, 2015; Rest et al., 2016). However this is for the 434 first time that the tomato was selected as the target plant to detect genes involved in a wide range 435 of biotic and abiotic stress responses. In previous studies, usually one or a few stresses were 436 considered, but here, a wide range of stresses was studied simultaneously. We identified 1862 437 and 835 genes differentially regulated in response to biotic and abiotic stresses, respectively, and 438361 genes identified in this work are to be involved in cross-talk between biotic and abiotic stress responses. Since transcription factors regulate the expression of a wide range of genes under 440 stress conditions, we focused on genes which encode transcription factors and it was revealed 441 that close to $5 \%$ of identified genes encoding TFs. Among them, the expression of genes 442 encoding JERF1, GRAS6, MYB48, SlERF4, EIL2, protein LHY, SlERF1, WRKY 26, bZIP 443 transcription factor, ICE1-like, pti6, EIL3 and WRKY 11 are being affected by a diverse set of

444 biotic and abiotic stresses. This study highlighted the importance of detected TFs as regulators of 
445 various stress responses, reinforcing the role of these TFs in regulating plant tolerance to a board 446 spectrum of stresses (Borrás-Hidalgo et al., 2005; Yáñez et al., 2009; Sharma et al., 2010; Feng 447 et al., 2013; Li, Luan \& Liu, 2015). The results suggest that detected transcription factors in this 448 work play important roles in plant tolerance response to various stresses and data generated will 449 be helpful in conducting functional genomics studies to figure out their precise role during plant 450 stress response.

451 The salient features of this study include 1) a valuable approach to identify the expression 452 profiles of various genes under different conditions in plants; 2) meta-analysis can be used to 453 characterize candidate genes for both biotic and abiotic stress tolerance; 3) Identification of 454 important genes involved in various biotic and abiotic stresses; 4) we have identified genes 455 which are the potential candidates for genetic manipulation of plants to improve their tolerance 456 to biotic and abiotic stresses.

457

458 REFERENCES

459 Alves MS, Dadalto SP, Gonçalves AB, De Souza GB, Barros VA, Fietto LG. 2013. Plant bZIP 460 transcription factors responsive to pathogens: a review. International Journal of Molecular $461 \quad$ Sciences 14(4):7815-28 DOI 10.3390/ijms14047815.

462 Arie T, Takahashi H, Kodama M, Teraoka T. 2007. Tomato as a model plant for plant-pathogen 463 interactions. Plant Biotechnology 24(1):135-47 DOI doi.org/10.5511/plantbiotechnology.24. 464135.

465 Atkinson NJ, Lilley CJ, Urwin PE. 2013. Identification of genes involved in the response of 466 Arabidopsis to simultaneous biotic and abiotic stresses. Plant Physiology 162(4):2028-41 DOI $467 \quad 10.1104 /$ pp.113.222372. 
468 Berrocal-Lobo M, Molina A, Solano R. 2002. Constitutive expression of ETHYLENE-

469

470

471

472

473

474

475

476

477

478

479

480

481

482

483

484

485

486

487

488

489

490

491

492

493

494

495

496

497
RESPONSE-FACTOR1 in Arabidopsis confers resistance to several necrotrophic fungi. The Plant Journal 29(1):23-32 DOI 10.1046/j.1365-313x.2002.01191.x.

Borrás-Hidalgo O, Thomma BPHJ, Collazo C, Chacón O, Borroto CJ, Ayra C, Portieles R, López Y, Pujol M. 2005. EIL2 transcription factor and glutathione synthetase are required for defense of tobacco against tobacco blue mold. Molecular Plant-Microbe Interactions. 19(4):399-406 DOI https://doi.org/10.1094/MPMI-19-0399.

Borrás-Hidalgo O, Thomma BP, Collazo C, Chacón O, Borroto CJ, Ayra C, Portieles R, López Y, Pujol M. 2006. EIL2 transcription factor and glutathione synthetase are required for defense of tobacco against tobacco blue mold. Molecular Plant-Microbe Interactions 19(4):399-406 DOI 10.1094/ MPMI -19-0399.

Campain A, Yang YH. 2010. Comparison study of microarray meta-analysis methods. BMC Bioinformatics 11(1):408 DOI 10.1186/1471-2105-11-408.

Chakravarthy S, Tuori RP, D'Ascenzo MD, Fobert PR, Després C, Martin GB. 2003. The tomato transcription factor Pti4 regulates defense-related gene expression via GCC box and non-GCC box cis elements. The Plant Cell 1;15(12):3033-50 DOI doi.org/10.1105/tpc.017574.

Chang LC, Lin HM, Sibille E, Tseng GC. 2013. Meta-analysis methods for combining multiple expression profiles: comparisons, statistical characterization and an application guideline. $B M C$ Bioinformatics 14(1):368 DOI 10.1186/1471-2105-14-368.

Chinnusamy V, Ohta M, Kanrar S, Lee BH, Hong X, Agarwal M, Zhu JK. 2003. ICE1: a regulator of cold-induced transcriptome and freezing tolerance in Arabidopsis. Genes \& Development 17(8):1043-54 DOI 10.1101/gad.1077503.

Chen L, Song Y, Li S, Zhang L, Zou C, Yu D. 2012. The role of WRKY transcription factors in plant abiotic stresses. Biochimica et Biophysica Acta (BBA)-Gene Regulatory Mechanisms 1819(2):120-8 DOI 10.1016/j.bbagrm.2011.09.002.

Dou M, Fan S, Yang S, Huang R, Yu H, Feng X. 2016. Overexpression of AmRoseal gene confers drought and salt tolerance in rice. International Journal of Molecular Sciences 18(1):2 DOI 10.3390/ijms18010002.

Du Z, Zhou X, Ling Y, Zhang Z, Su Z. 2010. agriGO: a GO analysis toolkit for the agricultural community. Nucleic Acids Research 38(suppl_2):W64-70 DOI 10.1093/nar/gkq310.

PeerJ reviewing PDF | (2017:08:20219:2:0:CHECK 26 Feb 2018) 
498 Eulgem T, Somssich IE. 2007. Networks of WRKY transcription factors in defense signaling.

499 Current Opinion in Plant Biology 10(4):366-71 DOI 10.1016/j.pbi.2007.04.020.

500 Feng HL, Ma NN, Meng X, Zhang S, Wang JR, Chai S, Meng QW. 2013. A novel tomato MYC-

501 type ICE1-like transcription factor, SIICE1a, confers cold, osmotic and salt tolerance in

502 transgenic tobacco. Plant Physiology and Biochemistry 73:309-20 DOI 10.1016/j.plaphy .2013 $503 \quad .09 .014$.

504 Fisher RA. 1992. Statistical methods for research workers. London: Oliver and Boyd.

505 Fujita M, Fujita Y, Noutoshi Y, Takahashi F, Narusaka Y, Yamaguchi-Shinozaki K, Shinozaki 506 K. 2006. Crosstalk between abiotic and biotic stress responses: a current view from the points 507 of convergence in the stress signaling networks. Current Opinion in Plant Biology 9(4):436-42 508 DOI 10.1016/j.pbi.2006.05.014.

509 Grundy J, Stoker C, Carré IA. 2015. Circadian regulation of abiotic stress tolerance in plants. $510 \quad$ Frontiers Plant Science 6:648 DOI 10.3389/fpls.2015.00648.

511 Gu YQ, Wildermuth MC, Chakravarthy S, Loh YT, Yang C, He X, Han Y, Martin GB. 2002. 512 Tomato transcription factors Pti4, Pti5, and Pti6 activate defense responses when expressed in 513 Arabidopsis. The Plant Cell 14(4):817-31 DOI 10.1105/tpc.000794.

514 Huang D, Jaradat MR, Wu W, Ambrose SJ, Ross AR, Abrams SR, Cutler AJ. 2007. Structural 515 analogs of ABA reveal novel features of ABA perception and signaling in Arabidopsis. The 516 Plant Journal 50(3):414-28 DOI 10.1111/j.1365-313X.2007.03056.x.

517 Huang S, Gao Y, Liu J, Peng X, Niu X, Fei Z, Cao S, Liu Y. 2012. Genome-wide analysis of 518 WRKY transcription factors in Solanum lycopersicum. Molecular Genetics and Genomics 519 287(6):495-513 DOI 10.1007/s00438-012-0696-6.

520 Huang XS, Zhang Q, Zhu D, Fu X, Wang M, Zhang Q, Moriguchi T, Liu JH. 2015. ICE1 of

521 Poncirus trifoliata functions in cold tolerance by modulating polyamine levels through 522 interacting with arginine decarboxylase. Journal of Experimental Botany 66(11):3259-74 DOI $52310.1093 / \mathrm{jxb} / \mathrm{erv} 138$.

524 Hummel M, Rahmani F, Smeekens S, Hanson J. 2009. Sucrose-mediated translational control. 525 Annals of Botany 104(1):1-7 DOI 10.1093/aob/mcp086.

526 Kim JG, Stork W, Mudgett MB. 2013. Xanthomonas type III effector XopD desumoylates 527 tomato transcription factor SIERF4 to suppress ethylene responses and promote pathogen 528 growth. Cell Host \& Microbe 13(2):143-54 DOI 10.1016/j.chom.2013.01.006. 
529 Kissoudis C, Sunarti S, Van De Wiel C, Visser RG, van der Linden CG, Bai Y. 2016. Responses

530 to combined abiotic and biotic stress in tomato are governed by stress intensity and resistance

531 mechanism. Journal of Experimental Botany 67(17):5119-32 DOI 10.1093/jxb/erw285.

532 Khatri P, Drăghici S. 2005. Ontological analysis of gene expression data: current tools, 533 limitations, and open problems. Bioinformatics 21(18):3587-95 DOI 10.1093/bioinformatics/ 534 bti565.

535 Knapp S, Bohs L, Nee M, Spooner DM. 2004. Solanaceae - a model for linking genomics with 536 biodiversity. Comparative and Functional Genomics 5(3):285-91 DOI 10.1002/cfg.393.

537 Li S, Fu Q, Chen L, Huang W, Yu D. 2011. Arabidopsis thaliana WRKY25, WRKY26, and 538 WRKY33 coordinate induction of plant thermotolerance. Planta 233(6):1237-52 DOI $539 \quad 10.1007 / \mathrm{s} 00425-011-1375-2$.

540 Li JB, Luan YS, Jin H. 2012. The tomato SIWRKY gene plays an important role in the 541 regulation of defense responses in tobacco. Biochemical and Biophysical Research 542 Communications 427(3):671-6 DOI 10.1016/j.bbrc.2012.09.120.

543 Li D, Fu F, Zhang H, Song F. 2015. Genome-wide systematic characterization of the bZIP 544 transcriptional factor family in tomato (Solanum lycopersicum L.). BMC Genomics 16(1):771 545 DOI 10.1186/s12864-015-1990-6.

546 Li JB, Luan YS, Liu Z. 2015. SpWRKY1 mediates resistance to Phytophthora infestans and 547 tolerance to salt and drought stress by modulating reactive oxygen species homeostasis and 548 expression of defense-related genes in tomato. Plant Cell, Tissue and Organ Culture. 549 123(1):67-81 DOI 10.0.3.239/s11240-015-0815-2.

550 Liu B, Ouyang Z, Zhang Y, Li X, Hong Y, Huang L, Liu S, Zhang H, Li D, Song F. 2014. 551 Tomato NAC transcription factor SISRN1 positively regulates defense response against biotic 552 stress but negatively regulates abiotic stress response. PLoS One 9(7):e102067 DOI 553 10.1371/journal.pone.0102067.

554 Lu SX, Knowles SM, Andronis C, Ong MS, Tobin EM. 2009. CIRCADIAN CLOCK 555 ASSOCIATED1 and LATE ELONGATED HYPOCOTYL function synergistically in the 556 circadian clock of Arabidopsis. Plant Physiology 150(2):834-43 DOI 10.1104/pp. 108. 133 557272. 
558 Mayrose M, Ekengren SK, Melech-Bonfil S, Martin GB, Sessa G. 2006. A novel link between 559 tomato GRAS genes, plant disease resistance and mechanical stress response. Molecular Plant 560 Pathology 7(6):593-604 DOI 10.1111/J.1364-3703.2006.00364.X.

561 Oh S, Park S, Han KH. 2003. Transcriptional regulation of secondary growth in Arabidopsis 562 thaliana. Journal of Experimental Botany 54(393):2709-22 DOI 10.1093/jxb/erg304.

563 Ouyang B, Yang T, Li H, Zhang L, Zhang Y, Zhang J, Fei Z, Ye Z. 2007. Identification of early 564 salt stress response genes in tomato root by suppression subtractive hybridization and microarray 565 analysis. Journal of Experimental Botany 58(3):507-20 DOI 10.1093/jxb/erl258.

566 Pan YJ, Cho CC, Kao YY, Sun CH. 2009. A novel WRKY-like protein involved in 567 transcriptional activation of cyst wall protein genes in Giardia lamblia. Journal of Biological 568 Chemistry 284(27):17975-88 DOI 10.1074/jbc.M109.012047.

569 Prasad KV, Abdel-Hameed AA, Xing D, Reddy AS. 2016. Global gene expression analysis 570 using RNA-seq uncovered a new role for SR1/CAMTA3 transcription factor in salt stress. 571 Scientific Reports 6:27021 DOI 10.1038/srep27021.

572 Rastgoo L, Alemzadeh A. 2011. Biochemical Responses of Gouan ('Aeluropus littoralis') to 573 Heavy Metals Stress. Australian Journal of Crop Science 5(4):375-83.

574 Rastgoo L, Alemzadeh A, Afsharifar A. 2011. Isolation of two novel isoforms encoding zinc-and 575 copper-transporting P1B-ATPase from Gouan (Aeluropus littoralis). Plant Omics Journal 4(7):377-83.

577 Rest JS, Wilkins O, Yuan W, Purugganan MD, Gurevitch J. 2016. Meta-analysis and metaregression of transcriptomic responses to water stress in Arabidopsis. The Plant Journal. 85(4): 548-560 DOI 10.1111/tpj.13124.

580

Rhodes DR, Barrette TR, Rubin MA, Ghosh D, Chinnaiyan AM. 2002. Meta-analysis of 581 microarrays: interstudy validation of gene expression profiles reveals pathway dysregulation in prostate cancer. Cancer Research 62(15):4427-33.

Roy S. 2016. Function of MYB domain transcription factors in abiotic stress and epigenetic control of stress response in plant genome. Plant Signaling \& Behavior 11(1):e1117723 DOI 10.1080/15592324.2015.1117723.

586

Sami Z, Alemzadeh A. 2016. Isolation and molecular characterization of a novel $\mathrm{Na}^{+} / \mathrm{H}^{+}$ antiporter gene, AlNHX2, from Aeluropus littoralis and comparison of AlNHX1 and AlNHX2. Plant Omics 9(3):205-12. 
589 Seki M, Narusaka M, Ishida J, Nanjo T, Fujita M, Oono Y, Kamiya A, Nakajima M, Enju A, 590 Sakurai T, Satou M. 2002. Monitoring the expression profiles of 7000 Arabidopsis genes under 591 drought, cold and high-salinity stresses using a full-length cDNA microarray. The Plant 592 Journal 31(3):279-92 DOI 10.1046/j.1365-313X.2002.01359.x.

593 Seo PJ, Mas P. 2015. STRESSing the role of the plant circadian clock. Trends in Plant Science 594 20(4):230-7 DOI 10.1016/j.tplants.2015.01.001.

595 Shaar-Moshe L, Hübner S, Peleg Z. 2015. Identification of conserved drought-adaptive genes 596 using a cross-species meta-analysis approach. BMC Plant Biology, 15:111 DOI 597 https://doi.org/10.1186/s12870-015-0493-6.

598 Shaik R, Ramakrishna W. 2014. Machine learning approaches distinguish multiple stress 599 conditions using stress-responsive genes and identify candidate genes for broad resistance in 600 rice. Plant Physiology 164(1):481-95 DOI 10.1104/pp.113.225862.

601 Sharma MK, Kumar R, Solanke AU, Sharma R, Tyagi AK, Sharma AK. 2010. Identification, 602 phylogeny, and transcript profiling of ERF family genes during development and abiotic stress 603 treatments in tomato. Molecular Genetics and Genomics 284(6):455-75 DOI 10.1007/s00438604 010-0580-1.

605 Sharma R, De Vleesschauwer D, Sharma MK, Ronald PC. 2013. Recent advances in dissecting 606 stress-regulatory crosstalk in rice. Molecular Plant 6(2):250-60 DOI 10.1093/mp/sss147.

607 Song C, Tseng GC. 2014. Hypothesis setting and order statistic for robust genomic meta608 analysis. The Annals of Applied Statistics 8(2):777-800.

609 Szklarczyk D, Franceschini A, Wyder S, Forslund K, Heller D, Huerta-Cepas J, Simonovic M, 610 Roth A, Santos A, Tsafou KP, Kuhn M. 2014. STRING v10: protein-protein interaction 611 networks, integrated over the tree of life. Nucleic Acids Research 43(D1):D447-52 DOI 612 10.1093/nar/gku1003.

613 Tseng GC, Ghosh D, Feingold E. 2012. Comprehensive literature review and statistical 614 considerations for microarray meta-analysis. Nucleic Acids Research 40(9):3785-99 DOI $61510.1093 /$ nar/gkr1265.

616 Van der Ent S, Verhagen BW, Van Doorn R, Bakker D, Verlaan MG, Pel MJ, Joosten RG, 617 Proveniers MC, Van Loon LC, Ton J, Pieterse CM. 2008. MYB72 is required in early 618 signaling steps of rhizobacteria-induced systemic resistance in Arabidopsis. Plant Physiology 619 146(3):1293-304 DOI 10.1104/pp.107.113829. 
620 Wang X, Lin Y, Song C, Sibille E, Tseng GC. 2012. Detecting disease-associated genes with 621 confounding variable adjustment and the impact on genomic meta-analysis: With application to 622 major depressive disorder. BMC Bioinformatics. 13:52 DOI https://doi.org/10.1186/1471$623 \quad 2105-13-52$.

624 Wilkinson B. 1951. A statistical consideration in psychological research. Psychological Bulletin $62548(3): 156-8$.

$626 \mathrm{Wu} \mathrm{KL}$, Guo ZJ, Wang HH, Li J. 2005. The WRKY family of transcription factors in rice and 627 Arabidopsis and their origins. DNA Research 12(1):9-26.

628 Wu L, Chen X, Ren H, Zhang Z, Zhang H, Wang J, Wang XC, Huang R. 2007. ERF protein 629 JERF1 that transcriptionally modulates the expression of abscisic acid biosynthesis-related 630 gene enhances the tolerance under salinity and cold in tobacco. Planta 226(4):815-25 DOI $631 \quad 10.1007 / \mathrm{s} 00425-007-0528-9$.

632 Wu X, Shiroto Y, Kishitani S, Ito Y, Toriyama K. 2009. Enhanced heat and drought tolerance in 633 transgenic rice seedlings overexpressing OsWRKY11 under the control of HSP101 promoter. 634 Plant Cell Reports 28(1):21-30 DOI 10.1007/s00299-008-0614-x.

635 Xiong H, Li J, Liu P, Duan J, Zhao Y, Guo X, Li Y, Zhang H, Ali J, Li Z. 2014. Overexpression 636 of OsMYB48-1, a novel MYB-related transcription factor, enhances drought and salinity 637 638 639 tolerance in rice. PLoS One 9(3):e92913 DOI 10.1371/journal.pone.0092913.

Yáñez M, Cáceres S, Orellana S, Bastías A, Verdugo I, Ruiz-Lara S, Casaretto JA. 2009. An abiotic stress-responsive bZIP transcription factor from wild and cultivated tomatoes regulates stress-related genes. 28(10):1497-1507 DOI https://doi.org/10.1007/s00299-009-0749-4.

Ying S, Jing-Yu X, Bian-Yun Y, Ben-Zhong Z, Yun-Bo L. 2004. Influence of salt stress on expression of some genes involved in the ethylene signalling pathway in tomato seedlings. Chinese Journal of Agricultural Biotechnology 1(2):115-8 DOI 10.1079/CJB200422. microarray analysis of tomato roots showed defined responses to iron deficiency. $B M C$ Genomics 13(1):101-15 DOI 10.1186/1471-2164-13-101.

Zhang H, Huang Z, Xie B, Chen Q, Tian X, Zhang X, Zhang H, Lu X, Huang D, Huang R. 2004. 648 The ethylene-, jasmonate-, abscisic acid-and NaCl-responsive tomato transcription factor 650 JERF1 modulates expression of GCC box-containing genes and salt tolerance in tobacco. Planta 220(2):262-70 DOI 10.1007/s00425-004-1347-x. 
651 Zhang Z, Li F, Li D, Zhang H, Huang R. 2010. Expression of ethylene response factor JERF1 in 652 rice improves tolerance to drought. Planta 232(3):765-74 DOI 10.1007/s00425-010-1208-8.

653 Zinati Z, Alemzadeh A, KayvanJoo AH. 2016. Computational approaches for classification and 654 prediction of P-type ATPase substrate specificity in Arabidopsis. Physiology and Molecular 655 Biology of Plants 22(1):163-74 DOI 10.1007/s12298-016-0351-5.

656

657 Acknowledgements

658 The authors are thankful to Dr. Ahmad Tahmasebi for his invaluable assistance throughout this 659 study.

660

661 


\section{Table $\mathbf{1}$ (on next page)}

Transcription factors and their binding sites

Transcription factor and binding site for each, in biotic and abiotic stresses. TF: Transcription factor, TFBS: Transcription factor binding site. 


\begin{tabular}{|c|c|c|c|}
\hline TF family & $\begin{array}{l}\text { NO. of TF in } \\
\text { abiotic }\end{array}$ & $\begin{array}{l}\text { NO. of TF } \\
\text { in biotic }\end{array}$ & TFBS \\
\hline ARF & 1 & 3 & TGTCTC auxin response elements (AuxRE) \\
\hline BBR-BPC & 1 & - & (GA/TC) 8 and GAGA-binding \\
\hline bHLH & 3 & 9 & E-box (5'-CANNTG-3') \\
\hline $\mathrm{C} 2 \mathrm{H} 2$ & - & 9 & a DNA element that contains an AGCT core \\
\hline $\mathrm{C} 3 \mathrm{H}$ & - & 1 & Unknown \\
\hline ERF & 5 & 18 & GCC box is an $11 \mathrm{bp}$ sequence (TAAGAGCCGCC) \\
\hline EIL & 3 & 2 & $\begin{array}{lcc}\text { EIL2 } & \text { BS } & \text { in } \\
\text { (TTCAAGGGGGCATGTATCTTGAA) }\end{array}$ \\
\hline FAR1 & - & 1 & FBS for FHY3-FAR1 binding site \\
\hline GATA & - & 1 & WGATAR $(\mathrm{W}=\mathrm{T}$ or $\mathrm{A} ; \mathrm{R}=\mathrm{G}$ or $\mathrm{A})$ motifs \\
\hline GRAS & 1 & 7 & cis-element AATTT \\
\hline HSF & - & 3 & $\begin{array}{l}\text { that consists in a tandem of inverted repeats of the } \\
\text { sequence GAA, generating a perfect }\end{array}$ \\
\hline LBD & - & 1 & $\begin{array}{l}\text { HSE, TTCnnGAAnnTTC } \\
\text { core sequence CGGC }\end{array}$ \\
\hline MIKC_MADS & - & 1 & keratin-like coiled-coil domain \\
\hline $\begin{array}{l}\text { MYB and MYB } \\
\text { related }\end{array}$ & 2 & 8 & $\begin{array}{l}\text { (CNGTTR), (GKTWGTTR), TAACPy sequence (only } \\
\text { one AAC sequenc) and (GKTWGGTR; R, A or G; K, G or } \\
\text { T; W, A or T) and EE, AGATATTT }\end{array}$ \\
\hline NAC & 5 & 4 & $\begin{array}{l}\text { NAC recognition site (NACRS), NAC binding element } \\
\text { (SNBE) with a longer and variable } \\
\text { sequence[T/A]NN[C/T][T/C/G]TNNNNNNNA[A/C]GN[ } \\
\mathrm{A} / \mathrm{C} / \mathrm{T}][\mathrm{A} / \mathrm{T}] \text { ) }\end{array}$ \\
\hline NF-YB & - & 3 & CCAAT binding \\
\hline NF-YC & 1 & - & core nucleotide sequence CCAAT \\
\hline PHD & - & 1 & Unknown \\
\hline SPL proteins & - & 1 & SBP-box, TNCGTACAA \\
\hline TALE & 2 & 1 & Pbx:Meinox binding site (CTGTCAATCA) \\
\hline TCP & - & 2 & GGNCCCAC sequences and s $\mathrm{G}(\mathrm{T} / \mathrm{C}) \mathrm{GGNCCC}$ \\
\hline TIFY & - & 1 & Unknown \\
\hline
\end{tabular}




\begin{tabular}{lccl} 
VOZ & 1 & - & GCGTNx7ACGC \\
WRKY & 3 & 9 & W box (TTGACY; Y, C or T) \\
YABBY & - & 1 & WATNATW $(\mathrm{W}=\mathrm{T}$ or A; R $=\mathrm{G}$ or A) \\
ZIP and HD-ZIP & 3 & 6 & motif AATNATT \\
\hline
\end{tabular}

1

2 


\section{Table 2 (on next page)}

Detected transcription factors

Detected transcription factors are affected by a diverse set of biotic and abiotic stresses. 


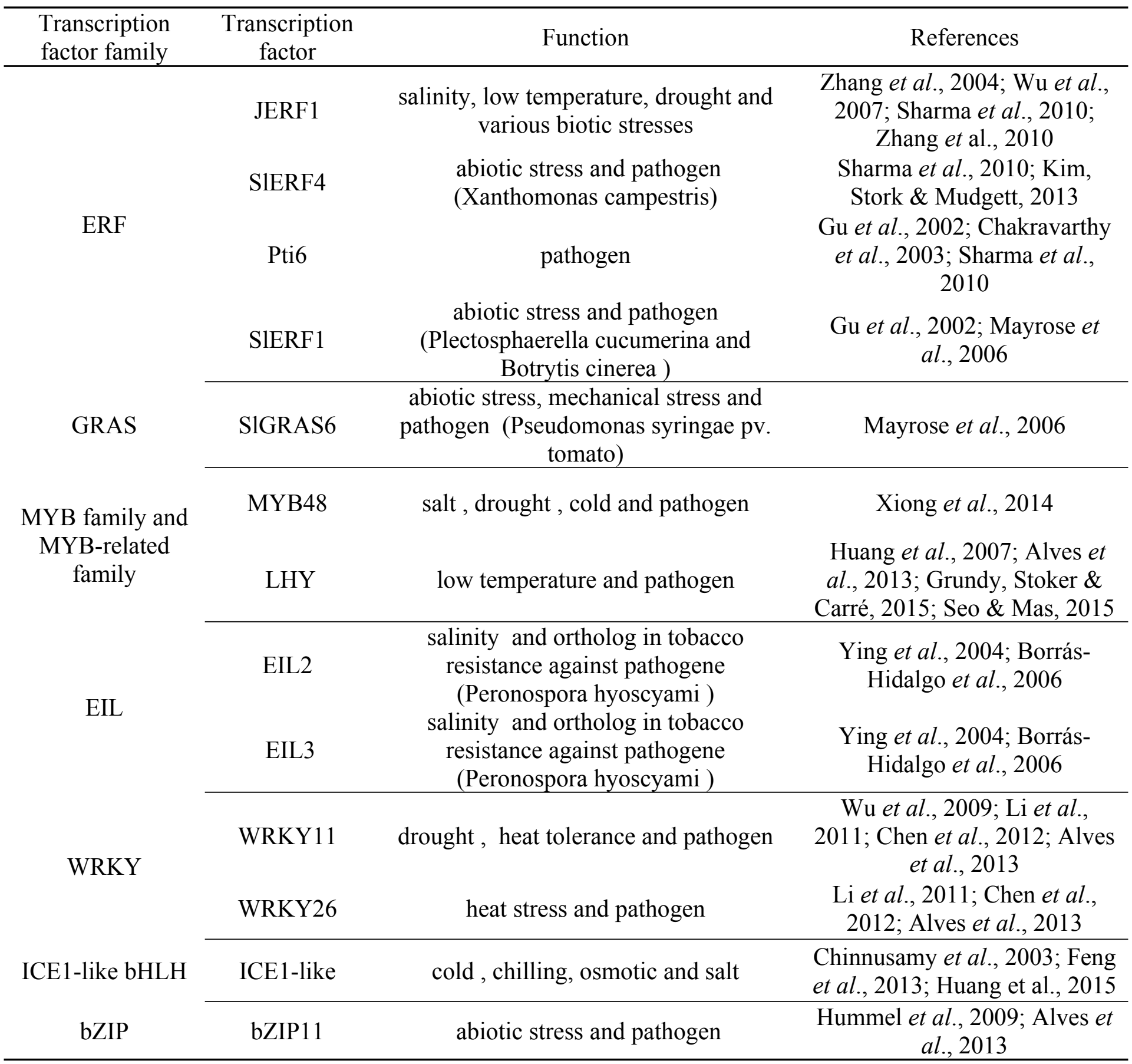




\section{Figure 1}

Identification of genes involved in biotic and abiotic stresses

Comparison of differentially expressed genes (DEGs) under abiotic and biotic stress responses, (A) Four-way Venn diagrams showing co-occurrence of DEGs in response to various abiotic and biotic stresses by two meta-analytical approaches: Fisher and maxP methods. (B) Four-way Venn diagrams showing number of transcription factors DEGs in all identified abiotic stresses by two different meta-analytical statistical methods: Fisher and maxP. (C) Four-way Venn diagrams showing number of transcription factors DEGs in all identified biotic stresses by two different meta-analytical statistical methods: Fisher and $\operatorname{maxP}$. 


\section{Abiotic maxP Biotic Fisher}

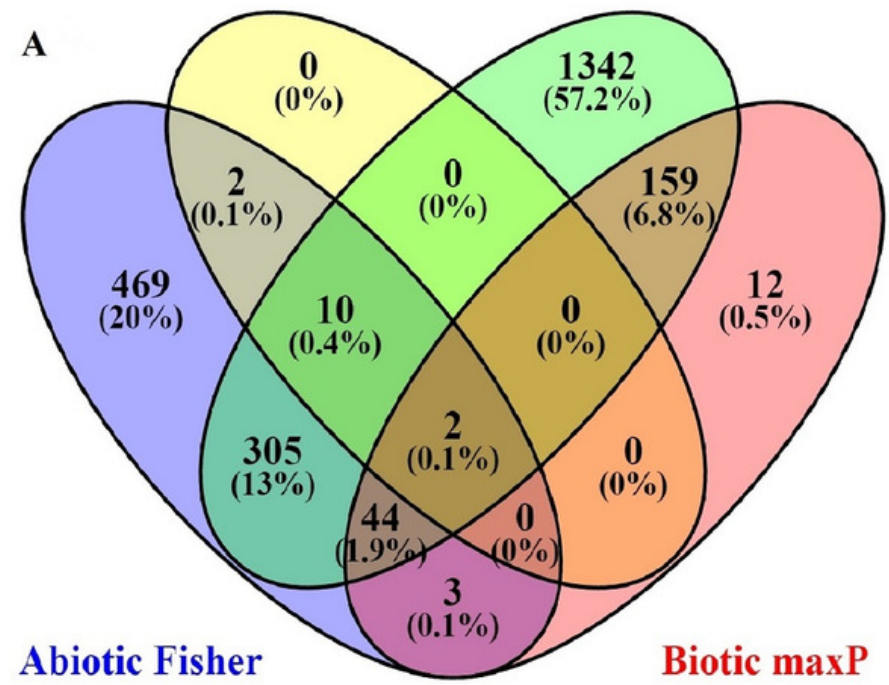

Ab. $\max P$

Ab. Fisher TF

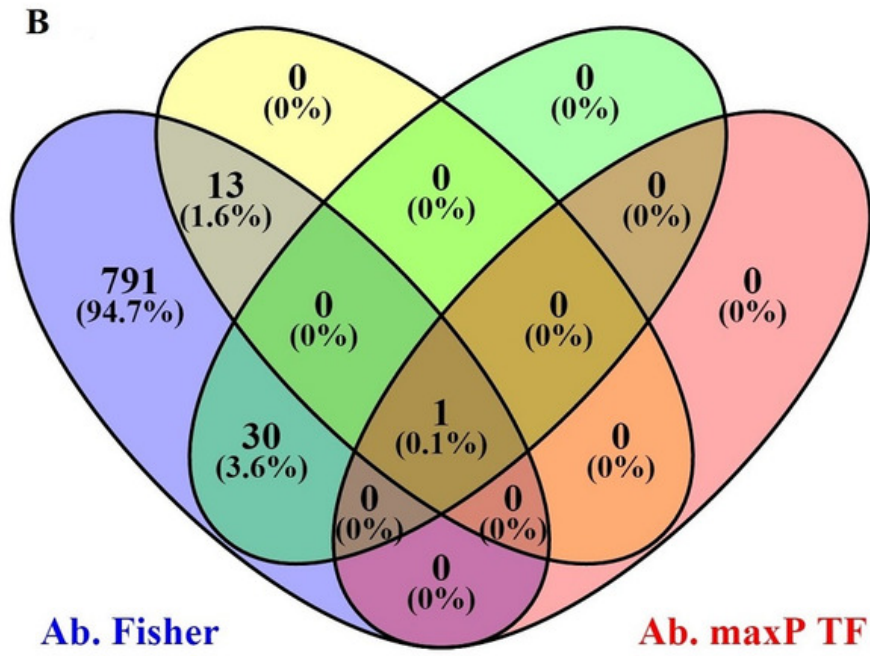

Bio. maxP

Bio. Fisher TF

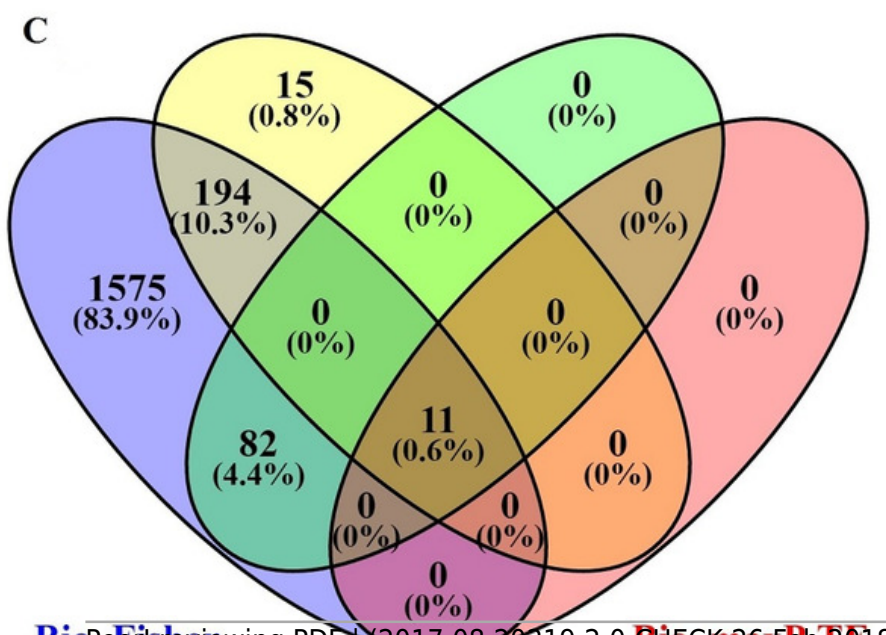




\section{Figure 2}

\section{Gene ontology}

Gene Ontology analysis. Frequency of most representative biological process terms. Com:

Differentially expressed genes obtained by common genes in biotic and abiotic stresses, AS:

Differentially expressed genes obtained by abiotic stresses, BS: Differentially expressed genes obtained by biotic stresses. BG: The frequency of these terms in the reference genes, Tomato Locus set. Gene Ontology analysis made in the AgriGO platform (FDR $=5 \%$ ). More details in Table S10 and Table S11. 


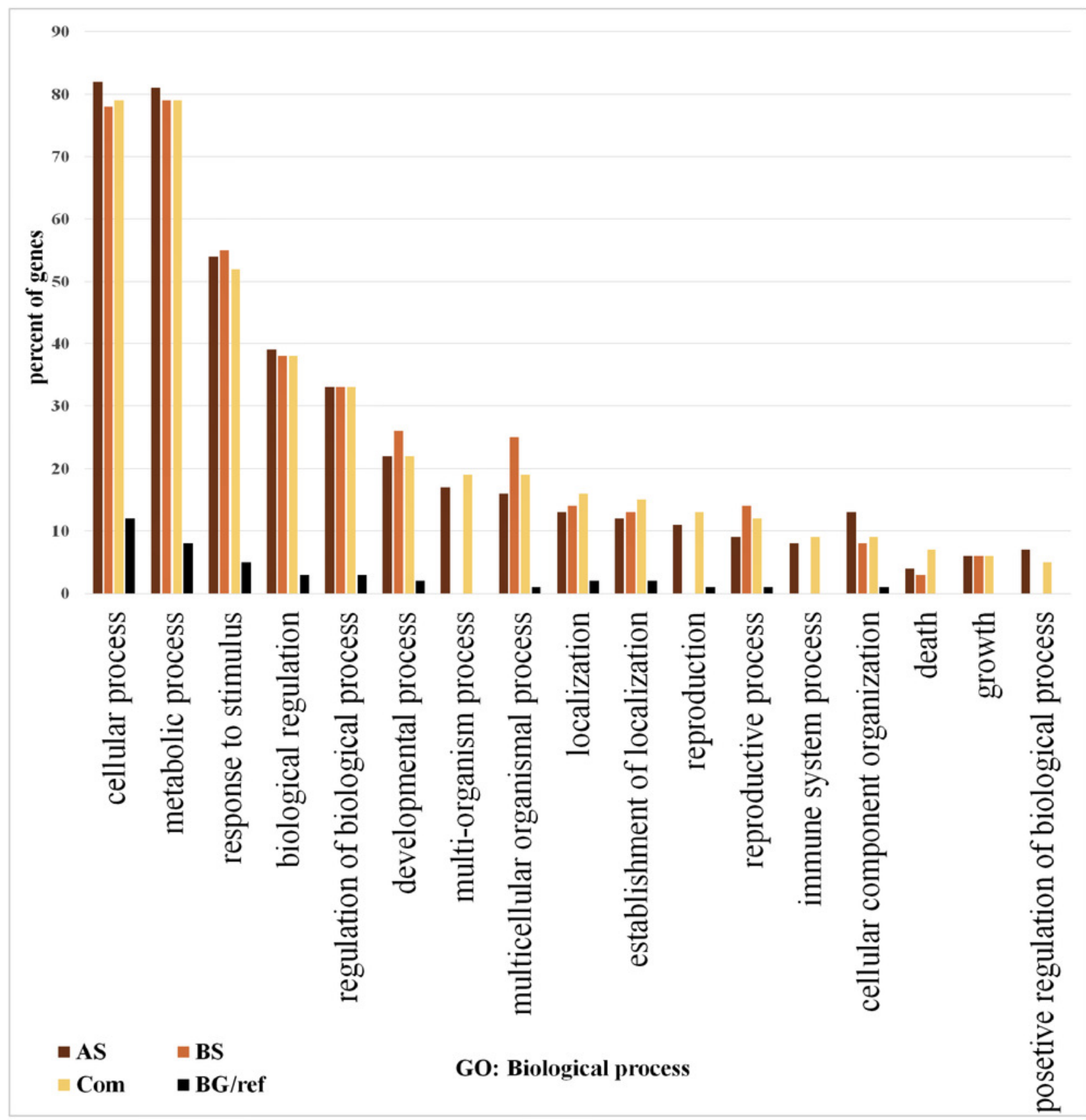




\section{Figure 3}

Network analysis of the DEGs identified in biotic and abiotic stresses

Network analysis of the 361 common DEGs identified in biotic and abiotic stresses. Network was generated by STRING (version 10.5) database and represents all connections of those genes with a confidence score $>0.4$. The connection colors show the types of evidence for concluding association: co-occurrence of those genes in the same organisms (dark blue), coexpression (black), experimental protein-protein interaction data (pink) and literature textmining (yellow). A number above hub represent TF. 1) JERF1, 2) MYB48, 3) protein LHY, 4) EIL3, 5) EIL2, 6) WRKY26. 


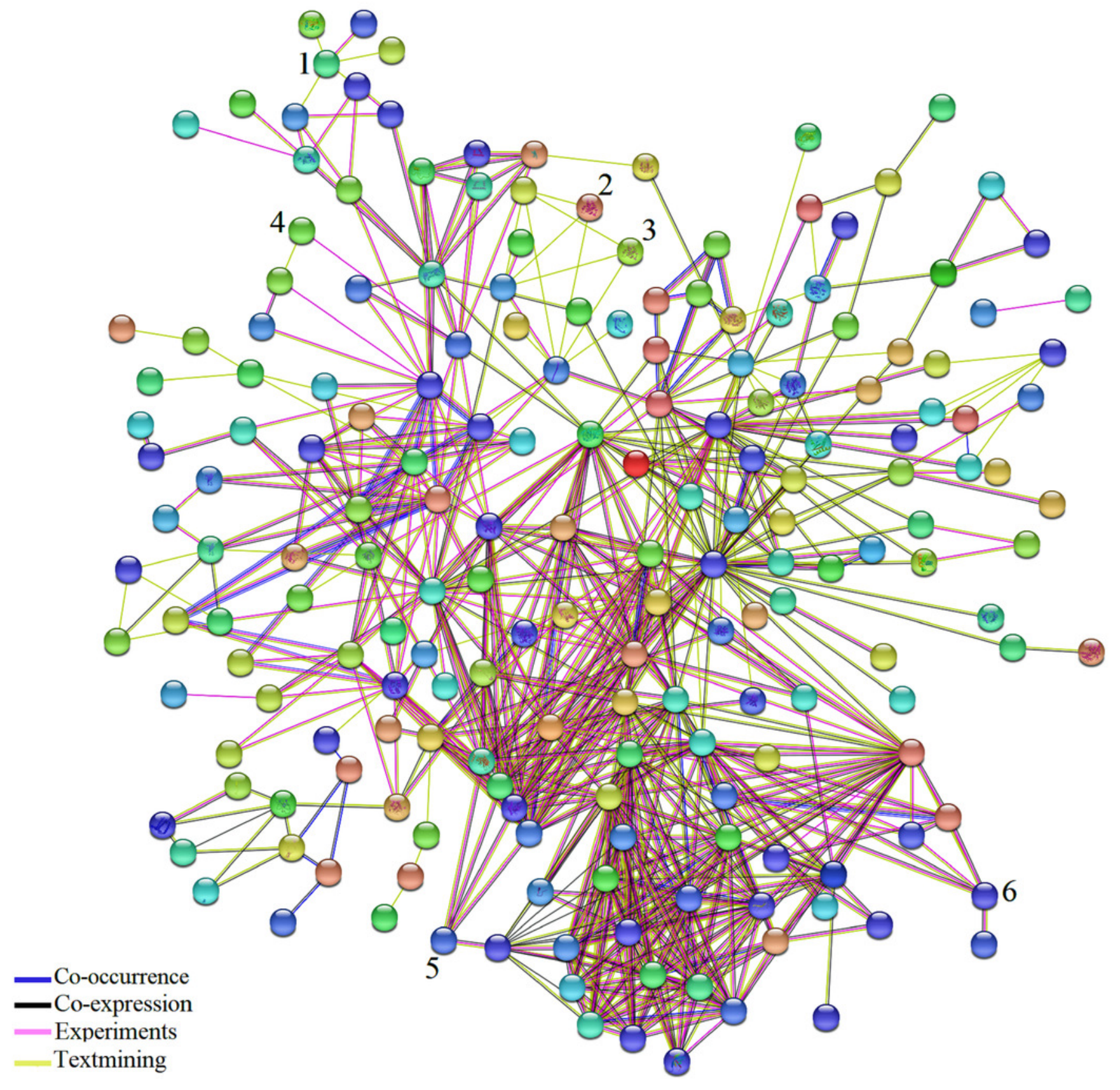




\section{Figure 4}

Common transcription factors in biotic and abiotic stresses

Two-way Venn diagram showing the common transcription factors between abiotic and biotic stresses.

\section{Abiotic TF Biotic TF}

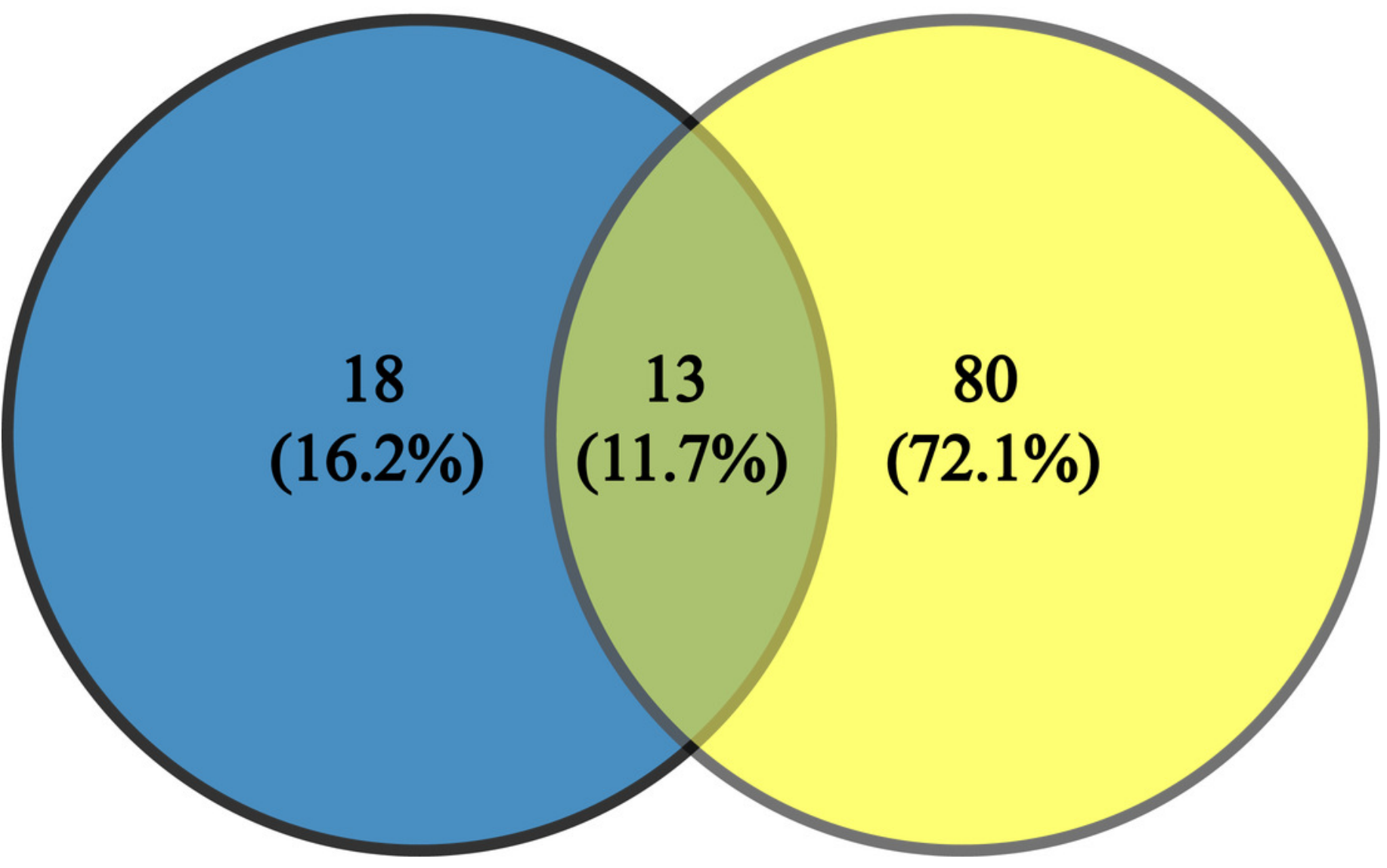


Figure 5

The presence or absence of the transcription factors across various organisms

Transcription factor occurrence patterns across various genomes. 1) EIL2, 2) EIL3, 3) ZIP, 4)

GRAS6, 5) SIERF4, 6) SIERF1, 7) JERF1, 8) WRKY26, 9) ICE1-like, 10) pti6, 11) WRKY11, 12)

protein LHY, 13) MYB48. The intensity of the color of the red square reflects the amount of

conservation of the homologous protein in the species. White color (No similarity detectable)

and black red color (100 similarity detectable).
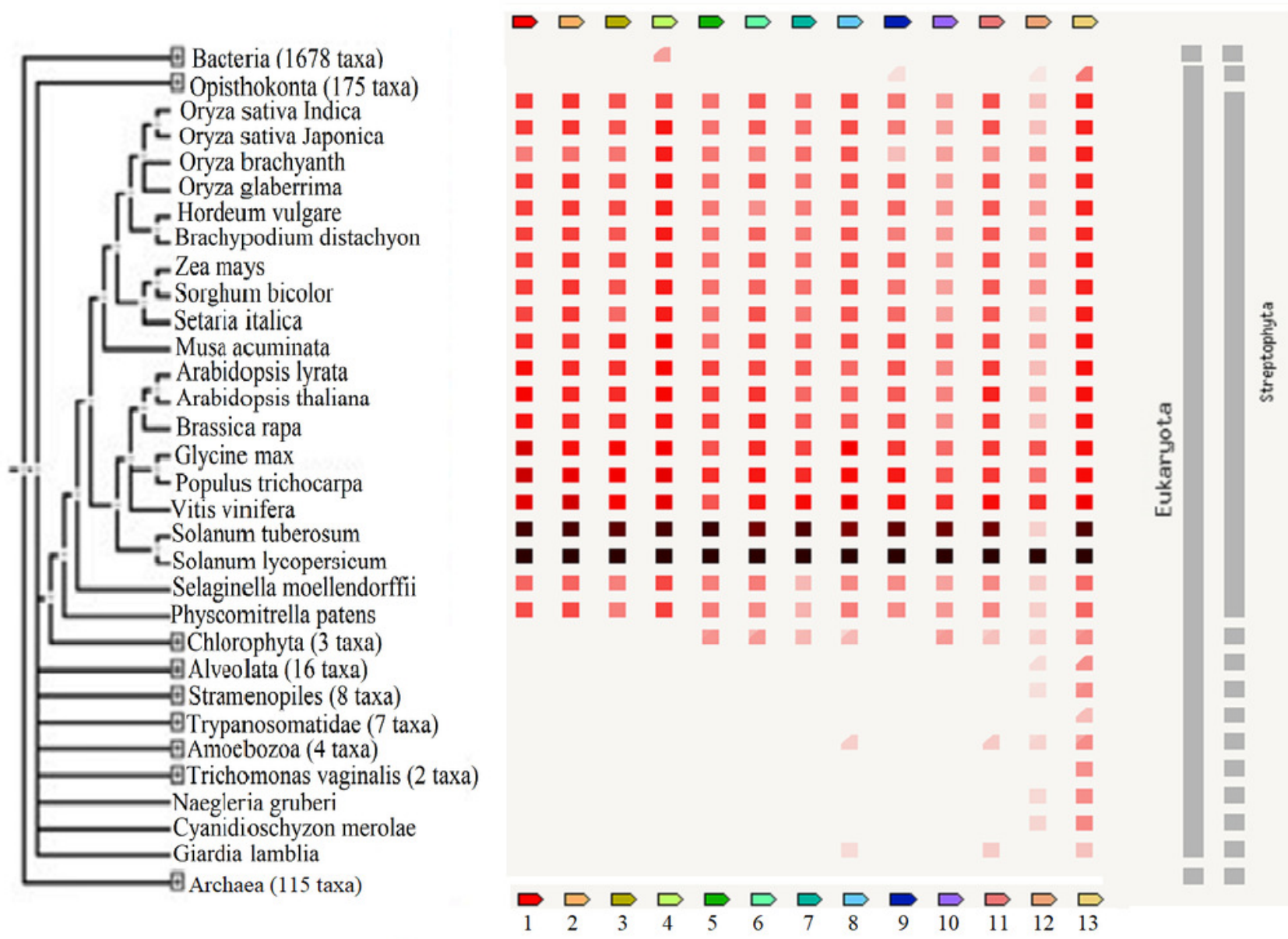